\title{
A new cross-layer scheme that combines grey relational analysis with multiple attributes and knapsack algorithms for WiMAX uplink bandwidth allocation
}

\author{
Ruey-Rong Su ${ }^{1,2^{*}}$, I-Shyan Hwang ${ }^{1}$ and Bor-Jiunn Hwang ${ }^{3}$
}

\begin{abstract}
To compensate for the adverse effects caused by radio link in worldwide interoperability for Microwave Access (WiMAX) networks, many radio resource management approaches have been present to assign orthogonal frequency division multiplexing access slots for seeking better performance. However, no WiMAX standards for optimal slot allocation have been defined. In this study, a new uplink bandwidth allocation scheme, GRAMA, which combines grey relational analysis with multiple attributes and the knapsack algorithms, is proposed to improve resource utilization while satisfying the user's requirements of throughput and fairness. Channel aware technology and service flow priority are used in the proposed scheme to achieve the highest performance index. A series of simulation is conducted under the scenarios of constant-bit-rate (CBR) voice traffic, variable-bit-rate (VBR) video traffic, and VBR data traffic. The performance is evaluated in terms of bandwidth utilization, transmission throughput, and fairness and simulation result which indicate that the proposed GRAMKA outperforms the conventional fuzzy and knapsack algorithms.
\end{abstract}

Keywords: Worldwide interoperability for Microwave Access (WiMAX), Uplink bandwidth allocation, Optimal slot allocation, Grey relational analysis with multiple attributes and knapsack algorithms (GRAMAKA), Fuzzy approach

\section{Introduction}

The mobile worldwide interoperability for Microwave Access (WiMAX) system is defined as a broadband wireless metropolitan area network (WMAN) in the IEEE 802.16e standard [1]. It aims to provide broadband wireless services anytime and anywhere by connecting mobile devices to base stations (BSs). In 2009, the WiMAX system was launched in Taiwan. WiMAX users in Taiwan numbered approximately 135,500 at the end of March 2013. Both orthogonal frequency division multiplexing (OFDM) and orthogonal frequency division multiplexing access (OFDMA) modulation technologies are used in the WiMAX system. In an OFDM system, a

\footnotetext{
* Correspondence: ronald@ukn.edu.tw

'Department of Computer Science and Engineering, Yuan-Ze University, Chung-Li 32003, Taiwan

${ }^{2}$ Department of Information Management, University of Kang Ning, Taipei 11485, Taiwan

Full list of author information is available at the end of the article
}

frame is composed of sub-channels with several subcarriers. Each sub-channel consists of symbols in the time domain [2] and sub-carriers in the frequency domain. The two types of data channel are the uplink channel (UL) in which data are sent from mobile station (MS) to the BS and the downlink channel (DL) in which data are sent from the BS to MS. These channels operate in a bandwidth that is distributed over $1.25-20 \mathrm{MHz}$, based on the available frequency resources and service demands. The WiMAX physical layer selects appropriate frames that are adapted to the time division duplex (TDD) mode for uplink and downlink directions to meet the user's demands. The OFDMA in mobile WiMAX systems is used to alleviate the adverse effect of multipath fading in non-line-of-sight (NLOS) environments. The OFDMA multiplexes multiple data streams onto DL and UL sub-channels [3]. In an OFDMA system, a data stream is divided into several parallel sub-streams with a reduced data rate (increasing the symbol 
duration), and each sub-stream is modulated and transmitted on a separate orthogonal sub-carrier. Five service types that are considered in mobile WiMAX systems are unsolicited grant service (UGS), real-time polling service (rtPS), extended rtPS (ertPS), non-real-time polling service (nrtPS), and best effort service (BES) [3]. In this work, to allocate the resources for data bursts [4], three services, voice service at a constant bit rate (CBR), video service at a variable bit rate (VBR), and the VBR data service, are considered. IEEE 802.16 defines burst profiles based on the combination of the modulation and coding schemes in each connection of physical configuration to adapt the data rate for the user. To compensate for the adverse effects of radio link fading, interference, and noise effects [4], many radio resource management (RRM) techniques have been developed, improving the efficiency and reliability of wireless transmission [5]. Channel aware technology enables a BS to choose the modulation and coding scheme based on the carrier-to-interference noise ratio (CINR) by link adaptation. In IEEE 802.16e, the CINR of every mobile station changes dynamically. Many RRM algorithms have been presented to assign OFDMA slots according to the channel conditions for meeting user's demand [6]. However, no WiMAX standard for optimal slot allocation has been defined.

Gupta and Chandavarkar [7] proposed a bandwidth management algorithm for WiMAX networks that provided reasonable performance, but it did not take the advantage of channel aware features [6]. A bandwidth request analysis mechanism [8] that focuses on rtPS and nrtPS traffic has been presented, but voice traffic (unsolicited granted service) was not considered. A radio resource allocation algorithm for uplink bandwidth allocation and recovery has been developed [9] to provide proportional and fair bandwidth utilization. The problems of the aforementioned methods are that the bandwidth is not allocated efficiently and the user's requirements are not well considered. A grant allocation algorithm, called half-duplex allocation (HDA), which was developed by Bacioccola et al. [10] aims to ensure a consistent and feasible grant allocation. However, the HDA algorithm does not efficiently allocate bandwidth because it exhibits poor throughput. An adaptive bandwidth allocation scheme (ABAS) is proposed by Chiang et al. [11] to vary the allocated bandwidth ratio according to the current traffic profiles. However, it does not consider service flow priority, resulting in poor throughput performance.

Grey relational analysis (GRA) [12] is an accurate predictor which needs smaller samples than required by conventional schemes, such as fuzzy and knapsack. The computational burden is thus significantly lower. Unlike the fuzzy [13] and knapsack algorithms [14], the GRA

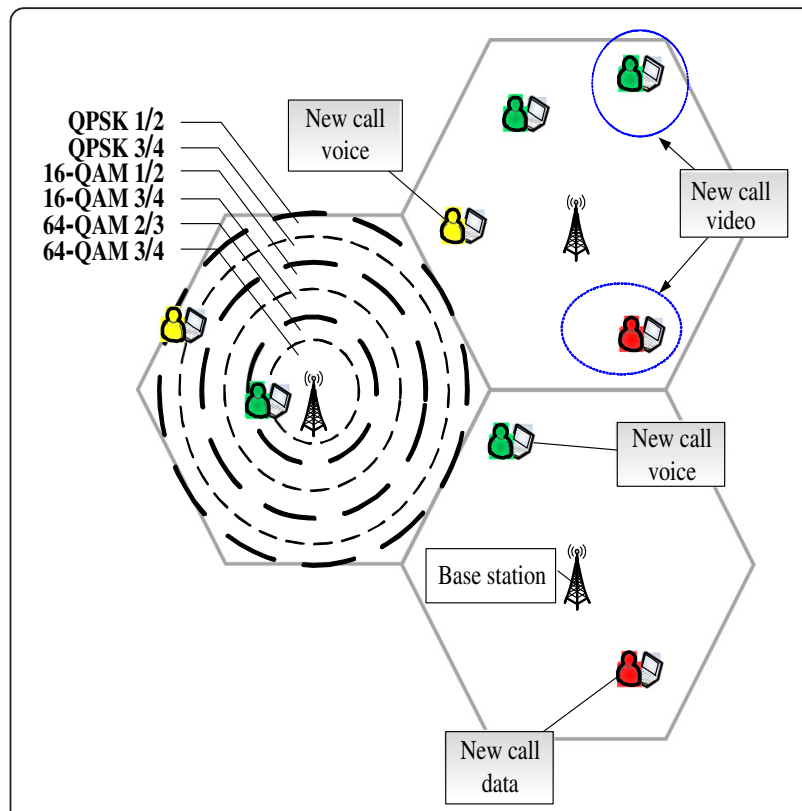

Fig. 1 System structure

yields consistent results in both quantitative and qualitative analysis. In this paper, a new uplink bandwidth allocation scheme, grey relational analysis with multiple attributes and knapsack algorithms (GRAMAKA), which combines GRA with multiple attributes [13] and the knapsack algorithm [14], is proposed to improve resource utilization while satisfying the user's requirements of throughput and fairness. The performance is evaluated in terms of bandwidth utilization, transmission throughput, and fairness. Channel aware technology [6] and service flow priority are applied in the proposed scheme to achieve the highest performance index. The rest of this paper is organized as follows. Section 2 elucidates the proposed GRAMAKA scheme. Section 3 presents the simulation results, and finally, Section 4 draws conclusions.

\section{Proposed GRAMAKA scheme}

Firstly, this section elucidates the structure of the system for simulation. Then, the fuzzy theory, the knapsack algorithm (KA), GRA, and the proposed GRAMAKA are described.

Table 1 System parameters

\begin{tabular}{ll}
\hline Parameter & Value \\
\hline Channel bandwidth & $10 \mathrm{MHz}$ \\
Peak uplink data rate & $28 \mathrm{Mbps}$ per sector in a 10 MHz channel \\
Frame duration & $5 \mathrm{~ms}$ \\
Maximum number of users & 600 \\
\hline
\end{tabular}




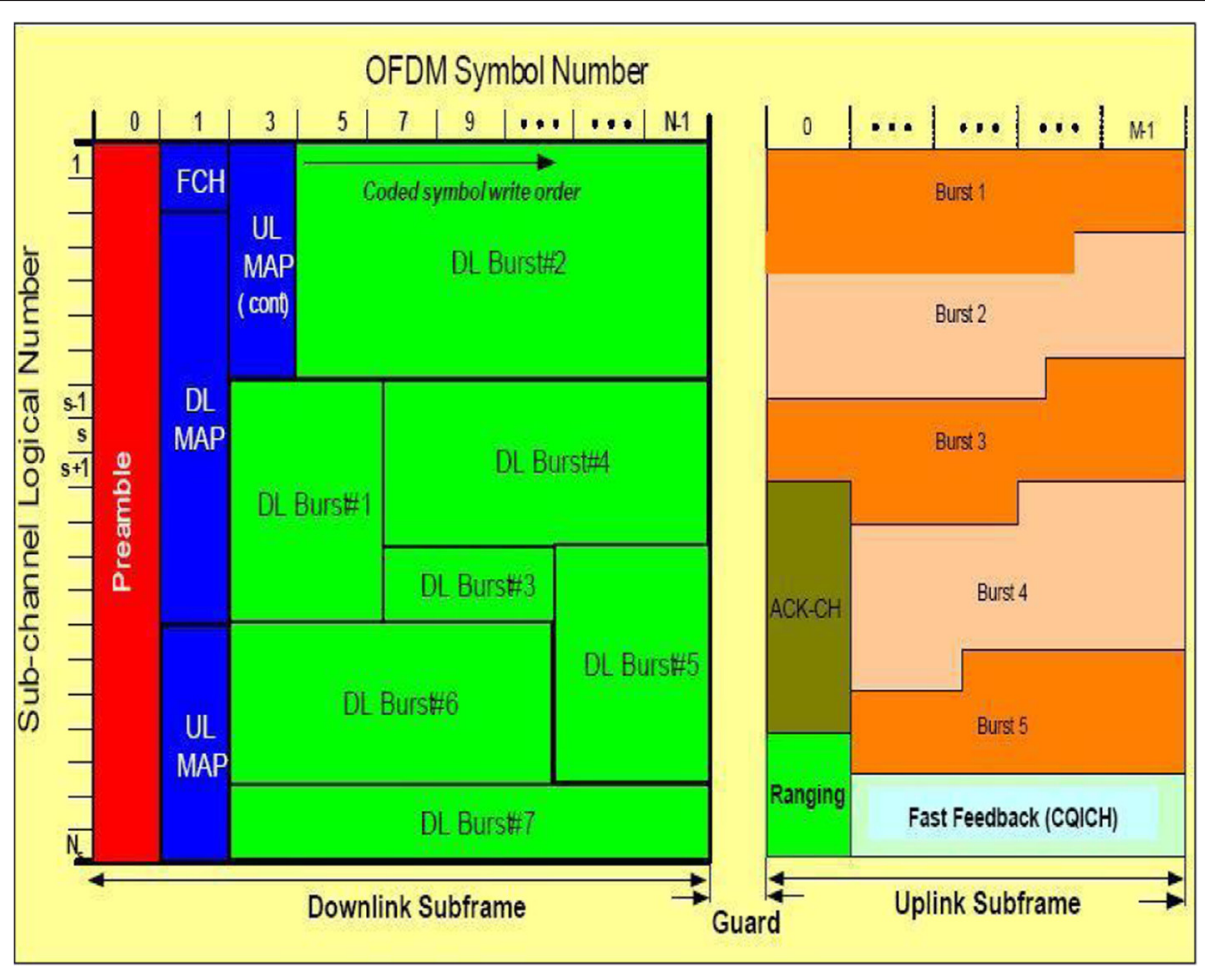

Fig. 2 WiMAX OFDMA and a frame structure

\subsection{System structure}

Figure 1 shows the structure of the system for simulation, a system that is used in the performance simulation, in which the simulation parameters are as given in Table 1 and OFDMA and TDD are used. In this environment, mobile users can access network resources using the WiMAX BS and may travel among the BSs; users may make new video calls to transmit video and new audio calls to transmit voice. When a new call arrives, the BS categorizes it using channel aware technology and assigns an optimal block [15] based on the signal to noise ratio (SNR). The range of code modulations is from QPSK $1 / 2$ to 64 QAM 3/4.

Figure 2 presents the WiMAX OFDM and a frame structure for TDD implementation. Each frame is divided into DL and UL sub-frames, which are separated by transmit/receive and receive/transmit transition gaps (TTG and RTG, respectively). Downlink MAP (DLMAP) and uplink MAP (UL-MAP) provide sub-channel allocation and other control information for the DL and

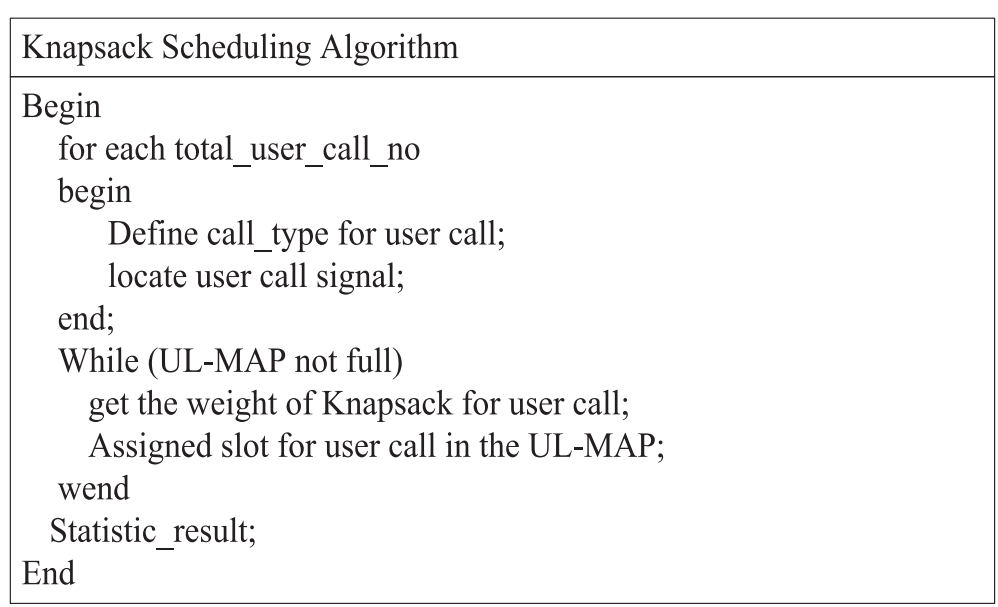

Fig. 3 Pseudocode of knapsack algorithm 


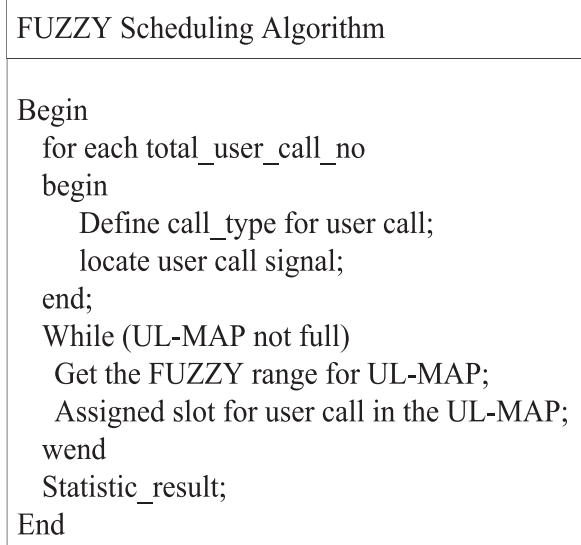

Fig. 4 Pseudocode of fuzzy approach

UL sub-frames. In this paper, the proposed radio resource allocation algorithm, GRAMAKA, for uplink bandwidth allocation is used to insert efficiently the slot of the resource block in UL-MAP as efficiently as possible.

\subsection{Fuzzy theory}

Fuzzy logic [13] is a form of many-valued logic, which supports reasoning that is approximate rather than fixed or exact. Whereas traditional binary sets (in which variables may have true or false values), fuzzy logic variables may have a truth value that ranges in degree between 0 and 1. Fuzzy logic has been extended to handle the concept of partial truth, where the truth value ranges between completely true and completely false. The values of these linguistic variables can be managed using specific functions, such as Trimf, Sigmf, and Gaussmf [16].

\subsection{Knapsack algorithm}

The knapsack problem [14] derives its name from the problem that is faced by someone who has a fixed-size knapsack and must fill it with the most useful items. It is a combinational optimization problem: given a set of items, each with a weight $w_{i}$ and a value $v_{i}$ determines the number of each item to be included in a collection so that the total weight is less than or equal to a given limit and the total value is as large as possible. All values and weights are usually assumed to be non-negative. The most common formulation is the $0-1$ knapsack problem, which restricts the number $x_{i}$ of each item to

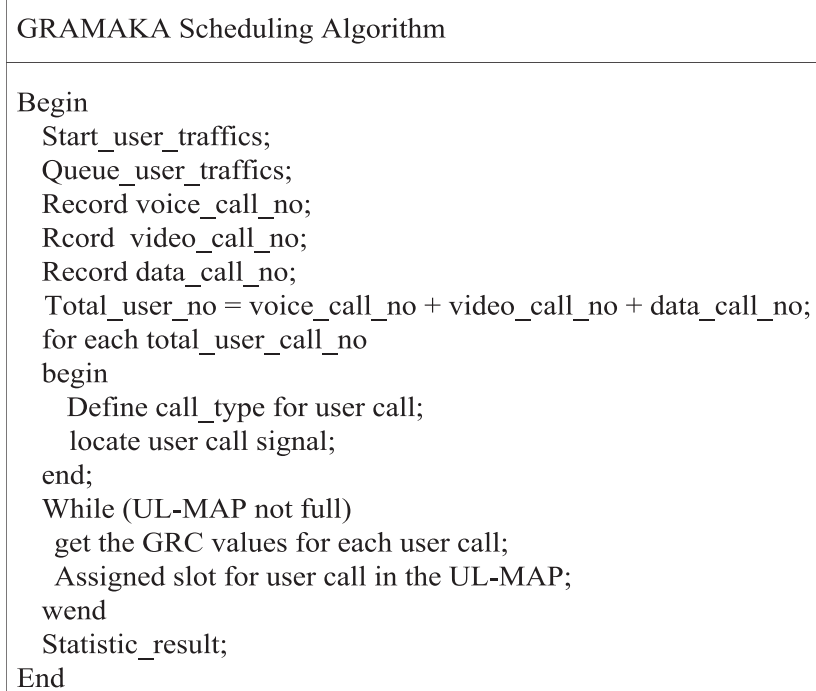




\section{Scheduling Algorithm}

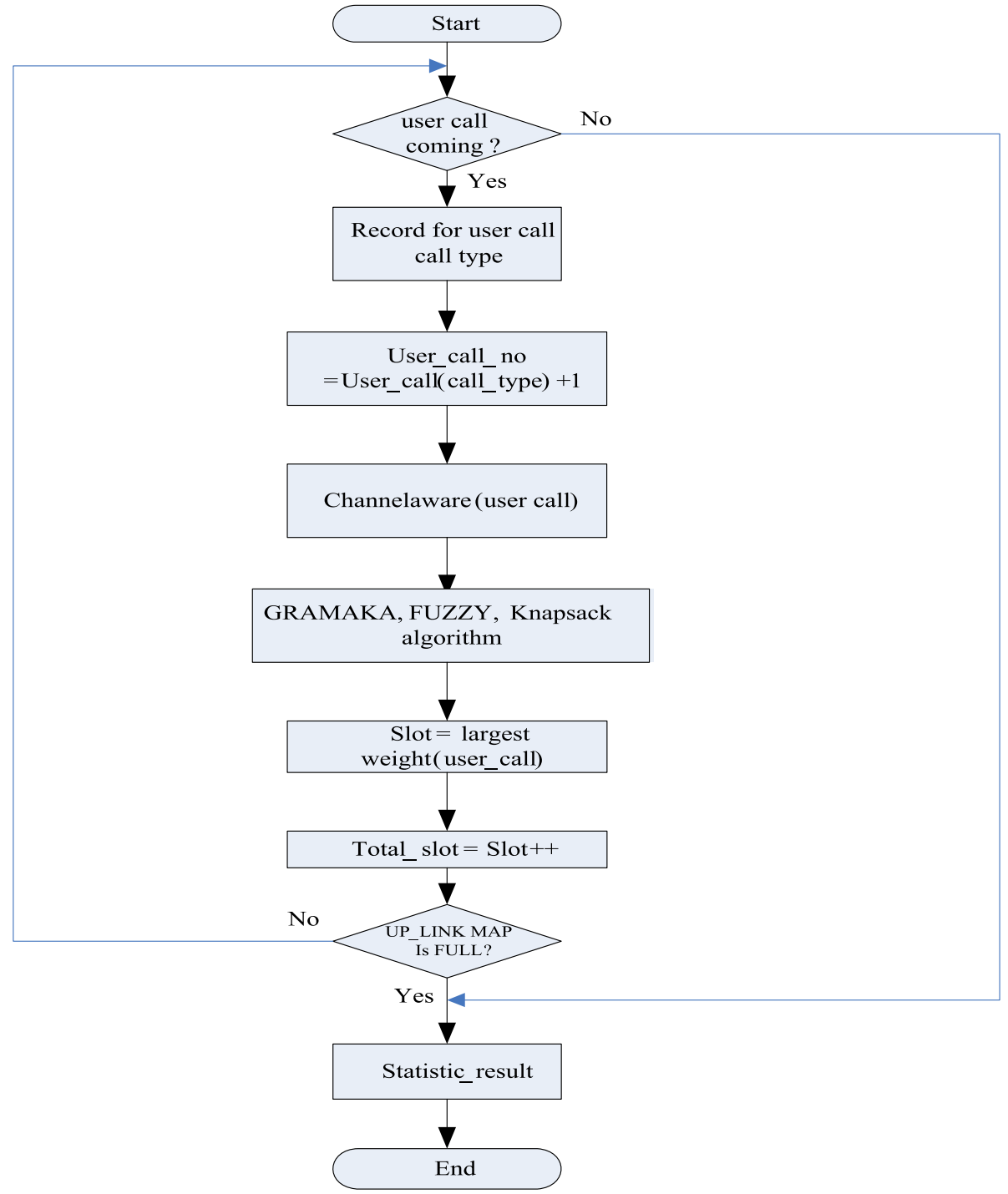

Fig. 6 Flow chart of scheduling algorithm

zero or one. Mathematically, the $0-1$ knapsack problem [14] is formulated as,

$$
\text { Maximize } \sum_{i=1}^{n} v_{i} x_{i} \text { subject to } \sum_{i=1}^{n} w_{i} x_{i} \leq W, x_{i} \in\{0,1\} .
$$

\subsection{GRA}

GRA [12] can be applied to the WiMAX resource allocation with the following definitions.

2.4.0.1 Definition 1: The influence space is denoted as $\{P\{x\}, Q\}$, with combination of the influence factors.
Weight $P\{x\}$ represents the number of OFDMA symbol, and value $Q$ denotes the number of sub-channel. The slots of UL-MAP are denoted as the original sequence $\mathrm{x}_{\mathrm{i}}^{(0)}(\mathrm{k})$ for user $i$, where $i=1,2, . ., N ; N$ is the maximum number of users.

$$
\mathbf{x}_{\mathbf{i}}^{(\mathbf{0})}(\mathbf{k})=\left(x_{1}^{(0)}(1), \ldots x_{i}^{(0)}(k)\right)
$$

where $k=1,2, \ldots, K . \mathbf{x}_{\mathbf{i}}^{(\mathbf{0})}(\mathbf{k})$ is used as a benchmark of sequence selection if the original sequence satisfies normalization, scaling, and polarization conditions. 
Table 2 Parameters of traffic types

\begin{tabular}{|c|c|c|c|c|}
\hline Traffic type & $\begin{array}{l}\text { Data rate } \\
\text { (kbps) }\end{array}$ & $\begin{array}{l}\text { Mean duration } \\
(\mathrm{sec})\end{array}$ & $\begin{array}{l}\text { Arrival rate } \\
\text { (call/s) }\end{array}$ & Traffic rate \\
\hline Voice services & 64 & 210 & $0.1-10$ & CBR \\
\hline Video services & 550-15550 & 360 & $0.1-10$ & VBR \\
\hline Data services & $280-7670$ & 180 & $0.1-10$ & VBR \\
\hline
\end{tabular}

2.4.0.2 Definition 2: The maximum slot's value denoted as

$$
\mathbf{x}_{\mathbf{i}}^{*}(\mathbf{k})=\left[\left(x_{i}^{(0)}(k) /\left(\max x_{i}^{(0)}(k)\right)\right]\right.
$$

$\mathbf{x}_{\mathbf{i}}^{*}(\mathbf{k})$ is a normalized value of the sequence of GRA with index $k=1,2, . . K$, and $\mathbf{x}_{\mathbf{i}}^{\mathbf{k}}(\mathbf{k})$ is the normalized value of sequence of GRA, where $1 \leq i \leq N$, with index $k=1,2, . . K$.

2.4.0.3 Definition 3: The minimum slot's value is denoted as

$$
\mathbf{x}_{\mathbf{i}}^{*}(\mathbf{k})=\left[\left(\min x_{i}^{(0)}(k)\right) /\left(x_{i}^{(0)}(k)\right)\right]
$$

where $1 \leq i \leq N$, with index $k=1,2, . ., K$, and $\mathbf{x}_{\mathbf{i}}^{*}(\mathbf{k})$ is the normalized value sequence of GRA.

2.4.0.4 Definition 4: The grey relational coefficient for the $i$ th combination of influence factors is denoted as

$$
\gamma\left(x_{i}(k), x_{j}(k)\right)=\left[\left(\Delta_{\min }+\varsigma \cdot \Delta_{\max }\right) /\left(\Delta_{i j}(k)+\varsigma \cdot \Delta_{\max }\right)\right]
$$

where $1 \leq i \leq N, j=1,2, \ldots J, k=1,2, \ldots K$, and $\zeta$ is a distinguishing coefficient. $x_{i}(k)$ is another set to be compared, where

$$
\Delta_{i j}(k)=\left|x_{i}(k)-x_{j}(k)\right|, \quad \Delta_{\min }=\min _{\forall j \in i} \min _{\forall k}\left\|x_{i}(k)-x_{j}(k)\right\|,
$$

and

$$
\Delta_{\max }=\max _{\forall j \in i} \max _{\forall k}\left\|x_{i}(k)-x_{j}(k)\right\| .
$$

Table 3 Throughput improvements obtained using fuzzy and GRAMAKA schemes over throughput obtained using knapsack algorithm, which serves as base line for voice traffic (CBR)

\begin{tabular}{lcl}
\hline Users number & Fuzzy (\%) & GRAMAKA (\%) \\
\hline 100 & 1.1 & 2.9 \\
200 & 1.4 & 3.9 \\
300 & 4.8 & 13 \\
400 & 6.6 & 17.9 \\
500 & 6.6 & 23.9 \\
600 & 11.2 & 29.9 \\
\hline
\end{tabular}

Table 4 Throughput improvements obtained using fuzzy and GRAMAKA schemes over throughput obtained using knapsack algorithm which provides base line for video traffic (VBR)

\begin{tabular}{lcc}
\hline Users number & Fuzzy (\%) & GRAMAKA (\%) \\
\hline 100 & 15.1 & 50.1 \\
200 & 15.6 & 56.9 \\
300 & 18.9 & 70.6 \\
400 & 18.9 & 72.9 \\
500 & 19.6 & 76.1 \\
600 & 29.1 & 79.2 \\
\hline
\end{tabular}

Based on the above definition, the steps of the execution of the GRA algorithm are as follows.

Step 1: GRA initializes the grey relational space $\{P\{x\}, Q\}$

Step 2: GRA modeling yields the local maximum and local minimum of the grey relational measure as,

$$
\mathrm{x}_{\mathrm{i}}^{*}(\mathrm{k})=\left[\left(x_{i}^{(0)}(k)-\min x_{i}^{(0)}(k)\right) /\left(\max x_{i}^{(0)}(k)-\min x_{i}^{(0)}(k)\right)\right]
$$

and

$$
\mathbf{x}_{\mathrm{i}}^{*}(\mathbf{k})=\left[\max \left(x_{i}^{(0)}(k)-x_{i}^{(0)}(k)\right) /\left(\max x_{i}^{(0)}(k)-\min x_{i}^{(0)}(k)\right)\right]
$$

Step 3: GRA coefficients are generated to obtained the grey relational coefficients of the $i$ th combination of influence factors as

$$
\gamma\left(x_{0}(k), x_{i}(k)\right)=\left[\left(\Delta_{\min }+\varsigma \cdot \Delta_{\max }\right) /\left(\Delta_{0 j}(k)+\varsigma \cdot \Delta_{\max }\right)\right]
$$

Step 4: Grey decision-making yields the priority of the selected sequence: a sequence with larger grey relational coefficients (GRC) has higher priority.

\subsection{Proposed GRAMAKA}

This work presents a new scheme, called GRAMAKA, which combines the GRA algorithm [12] with multiple attributes and knapsack algorithm. This optimization

Table 5 Throughput improvements obtained using fuzzy approach and GRAMAKA over throughput obtained using knapsack algorithm which provides base line for data traffic (VBR)

\begin{tabular}{lcc}
\hline Users number & Fuzzy (\%) & GRAMAKA (\%) \\
\hline 100 & 0.61 & 1.63 \\
200 & 0.61 & 1.63 \\
300 & 1.31 & 3.75 \\
400 & 1.59 & 5.04 \\
500 & 1.66 & 5.16 \\
600 & 1.89 & 6.04 \\
\hline
\end{tabular}




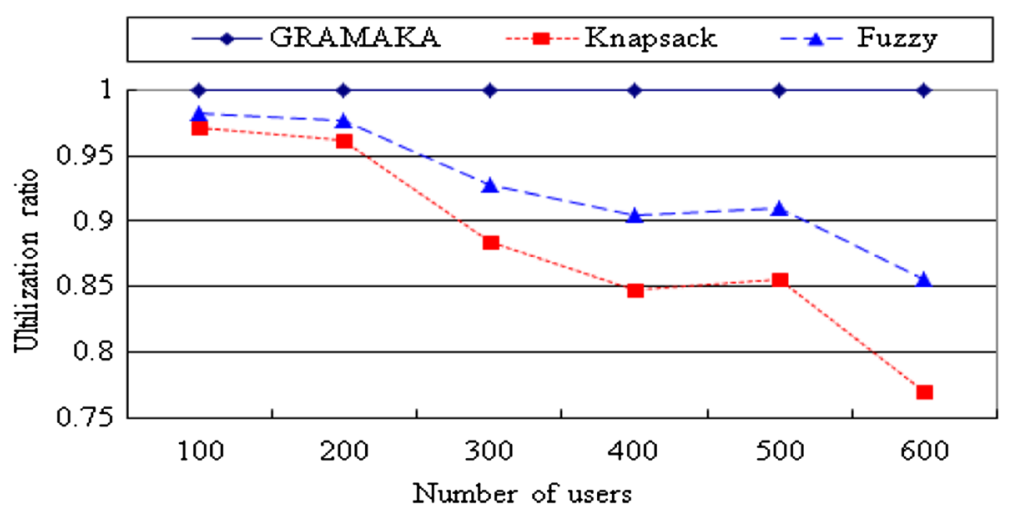

Fig. 7 Comparison of utilization ratios for voice traffic (CBR) that are obtained using knapsack, fuzzy, and GRAMAKA

scheme aims to find the best slot solution for the WiMAX's UL-MAP [17]. A detailed description of GRAMAKA is as follows. The length of the UL-MAP represents numbers of OFDMA symbol while the width denotes number of sub-channels. The utilization of resources that is represented by the area of the UL-MAP is maximized using channel aware technology [12]. The corresponding GRC of the $i$ th combination of influence factors is determined using Eq. (10) in step 3 of Section 2.4, as follows:

$$
\gamma\left(x_{0}(k), x_{i}(k)\right)=\left[\left(\Delta_{\min }+\varsigma \cdot \Delta_{\max }\right) /\left(\Delta_{0 j}(k)+\varsigma \cdot \Delta_{\max }\right)\right]
$$

where $x_{0}(k)$ is the reference sequence, $x_{i}(k)$ is the sequence to be compared with, $\Delta_{0 j}$ is the absolute value of the difference between $x_{0}(k)$ and $x_{i}(k)$, and $\varsigma \in[0,1]$ is the distinguishing coefficient. Finally, the grey decisionmaking process in step 4 in Section 2.4 yields the priority of the selected sequence. A sequence with a larger GRC has a higher priority being chosen as the best slots solution for allocating the resources of UL-MAP. Figure 2 presents the WiMAX OFDMA frame structure for UP-MAP.

\subsection{GRAMAKA scheduling algorithm}

Figures 3, 4, and 5 present the pseudocodes of the knapsack algorithm, the fuzzy approach, and the GRAMAKA scheme for scheduling, respectively.

Figure 6 presents the steps in executing the scheduling algorithms, which are as follows.

(1) If a user call is made, then record the type of call.

(2) Increase the number of the user call type.

(3) Process and record the user calls using the channel aware technology.

(4) Execute the GRAMAKA, fuzzy, and knapsack algorithms, respectively, for all user calls.

(5) Allocate the slots with the largest weights.

(6) Count the total number of slots for the user calls.

(7) If the total number of slots reaches the full size of the UL-MAP, then go to step (8); otherwise, go to step (1).

(8) Evaluate the simulation results and terminate the scheduling process.

\section{Simulation results}

A series of simulations is conducted using network simulator 2 (NS-2) [18] and the MATLAB tool, based on the system structure that is shown in Fig. 1, which is

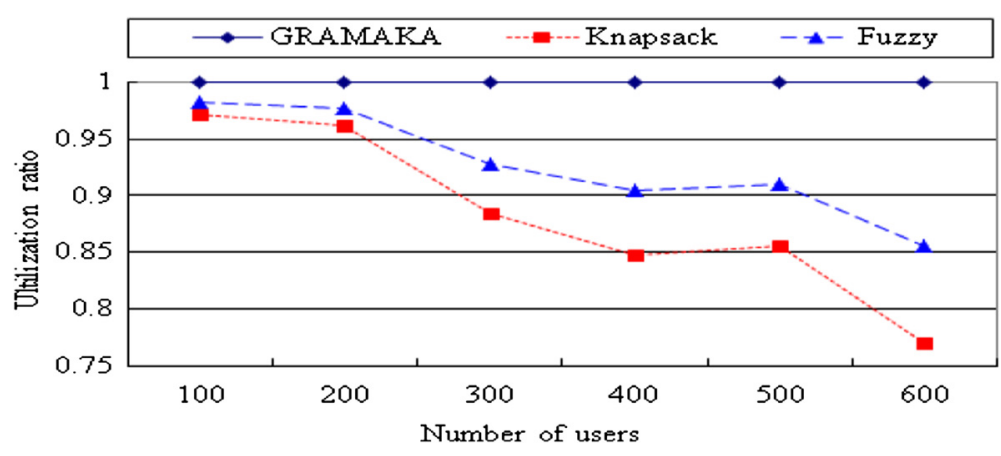

Fig. 8 Comparison of utilization ratios for video traffic (VBR) that are obtained using knapsack, fuzzy, and GRAMAKA 


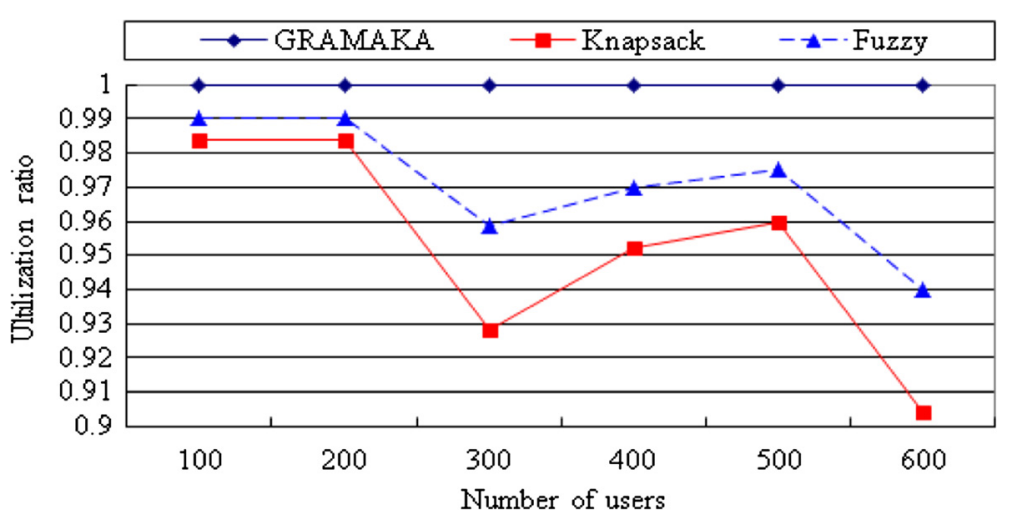

Fig. 9 Comparison of utilization ratios for data traffic (VBR) obtained using knapsack, fuzzy, and GRAMAKA

a two-ring structure with seven WiMAX cells. The BS in each cell is responsible for processing new calls. Mobile user calls with deferent burst profiles are random moves with a waypoint model [19] in each cell. In the simulation, the calls follow a Poisson distribution. The durations of the calls are exponentially distributed [20]. Tables 1 and 2 present the system parameters and traffic types [21], respectively. The slots of mobile users' channels have been adapted [22] effectively based on channel aware technology and the type of traffic [23]. The performances of the traditional knapsack, fuzzy, and the proposed GRAMAKA schemes have been evaluated and compared in terms of throughput and fairness. In the performance comparison, utilization ratio [24] is defined as the ratio of the utilized blocks to the capacity of the UL-MAP. Throughput is defined as the rate of successful data delivery over a communication channel. Throughput is usually measured in bits per second (bps). The performance index combines throughput with this fairness index. The fairness index is derived from Jane's fairness index [25] as $f\left(x_{1}, x_{2}, x_{3}, . ., x_{N}\right)=\frac{\left(\sum_{i=1}^{N} x_{i}\right)^{2}}{N \cdot \sum_{i=1}^{N} x_{i}^{2}}$

Jane's equation rates the fairness of a set of values where the number of users is $N$ and $x_{i}$ is the throughput of the $i$ th connection.

Table 3 presents the improvements in throughput that are obtained using the fuzzy algorithm and GRAMAKA scheme over the throughput that is obtained using the knapsack approach for voice traffic (CBR). The proposed GRAMAKA performed best, providing an improvement of 2.9-29.9\%, which is better than that, 1.1-11.2\%, achieved using the fuzzy algorithm.

Table 4 presents the throughput improvements that are achieved using the fuzzy algorithm and the GRAMAKA scheme over the throughput obtained knapsack approach for video traffic (VBR). The result demonstrates that the proposed GRAMAKA performed best,

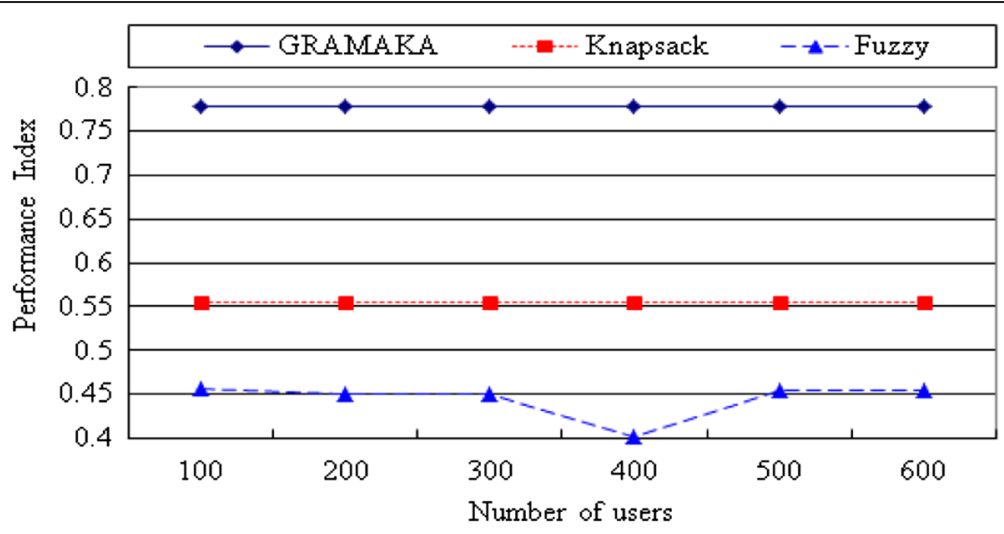

Fig. 10 Comparison of values of performance index obtained using knapsack, fuzzy, and GRAMAKA algorithms for voice traffic (CBR) 


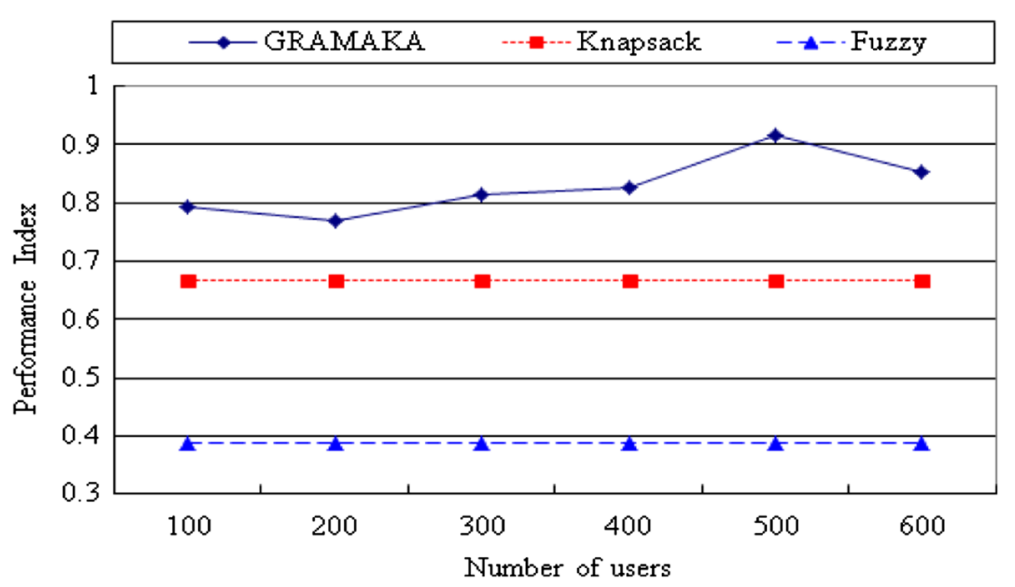

Fig. 11 Comparison of values of performance index obtained using different algorithms for video traffic (VBR)

providing an improvement of 50.1-79.2 \%, while the fuzzy algorithm provided an improvement of only $15.1-29.1 \%$.

Table 5 presents the throughput improvements that are achieved using the fuzzy algorithm and the GRAMAKA scheme over the throughput that is obtained using the knapsack approach for data traffic (VBR). The proposed GRAMAKA performed best. The GRAMAKA and fuzzy approach improved upon the knapsack approach by 1.63-6.04 and 0.61-1.89\%, respectively.

Figures 7, 8, and 9 compare the utilization ratios of voice traffic (CBR), video traffic (VBR), and data traffic (VBR) that are obtained using the knapsack, fuzzy, and proposed GRAMAKA schemes, respectively. The proposed GRAMAKA performs best in all scenarios because the combination of GRA with multiple attributes and the knapsack algorithm in response to throughput and fairness offers greater flexibility in the efficient insertion of the UL-MAP than do the other two approaches. Restated, multiple attributes properly deal with the throughput and fairness simultaneously. In contrast, the other two schemes have only one attribute, which allows them to address the throughput only. As shown by Figs. 7, 8, and 9, the efficient arrangement of the UL-MAP by the GRA decision-making, the GRAMAKA algorithm, has higher throughput than the other two algorithms and the utilization ratio of resources is thus significantly higher.

Figures 10, 11, and 12 compare the values of the performance index that are obtained using various algorithms for voice VBR data traffic, CBR voice traffic, and VBR video traffic. All results indicate that the proposed GRAMAKA is superior to the other two algorithms, owing to the flexibility of the insertion of slots into UL-MAP by GRA with multiple attributes.

Many related studies have considered different algorithms and environments, and thus, they are difficult to compare. Instead, Figs. 13, 14, and 15 are used to

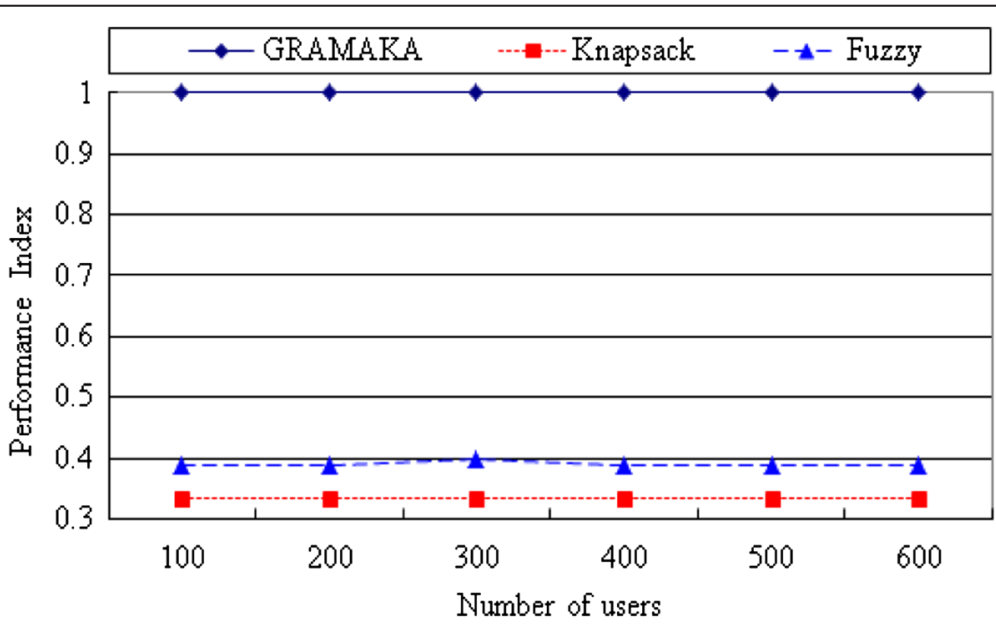

Fig. 12 Comparison of values of performance index obtained using different algorithms for data traffic (VBR) 


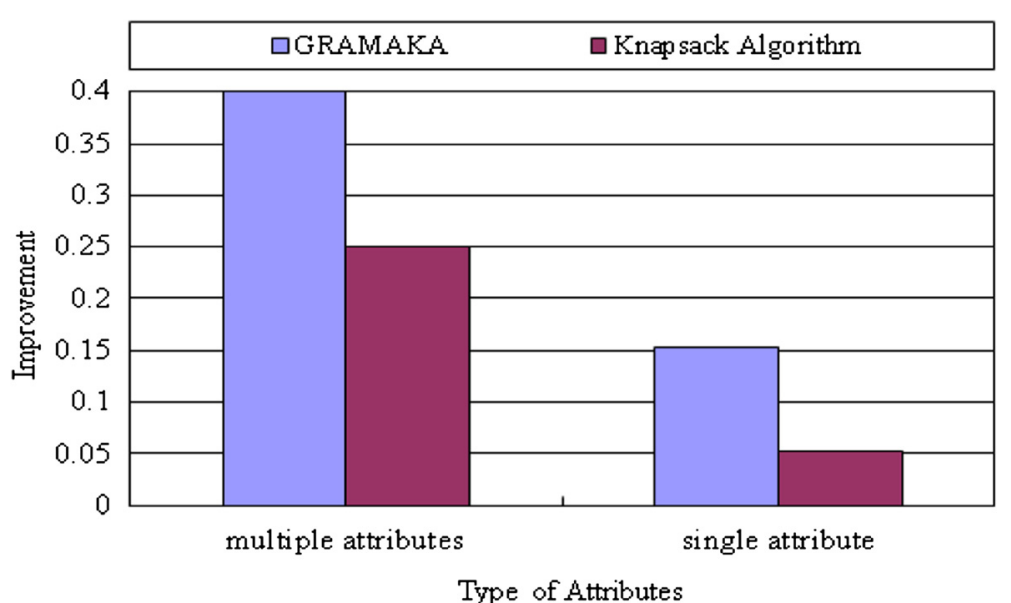

Fig. 13 Comparison of improvements for voice traffic (CBR) with different types of attributes

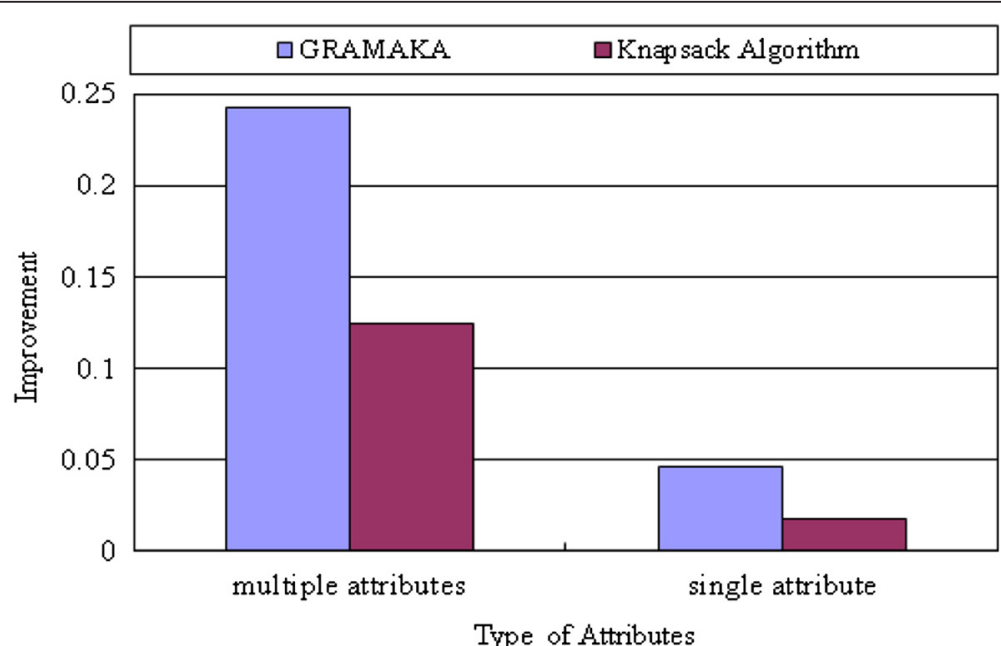

Fig. 14 Comparison of improvements for video traffic (VBR) with different types of attributes

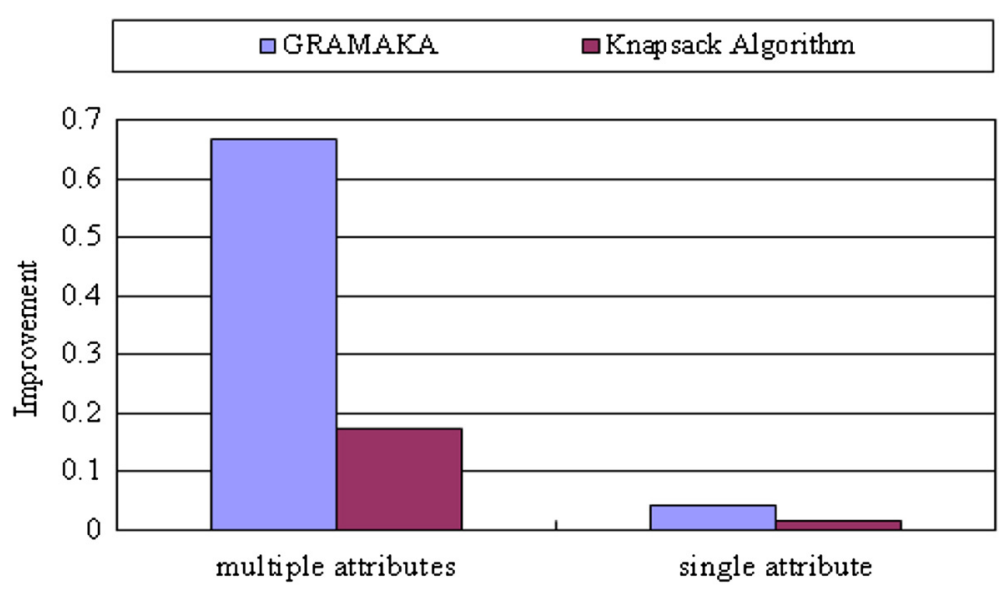

Type of Attributes

Fig. 15 Comparison of improvements for data traffic (VBR) with different types of attributes 
illustrate the throughput improvements that are obtained using GRAMAKA and knapsack algorithms with different types of attributes. All results show that the proposed GRAMAKA with multiple attributes performs best, offering a much greater improvement than the knapsack in cases of multiple attributes. In the case of a single attribute, the improvements achieved using GRAMAKA and knapsack algorithms are significantly lower.

\section{Conclusions}

A new scheme, GRAMAKA, which combines GRA with multiple attributes and the knapsack algorithms, has been proposed to improve resource utilization of the WiMAX networks. A series of simulations has been conducted using knapsack, fuzzy, and the proposed GRAMAKA algorithms under scenarios of constant-bitrate (CBR) voice traffic, variable-bit-rate (VBR) video traffic, and variable-bit-rate (VBR) data traffic, where the throughputs obtained using knapsack algorithm serve as benchmark for performance comparison. Simulation results demonstrate that the proposed GRAMAKA performed best under all scenarios. GRAMAKA provides improvements of 2.9-29.9, 50.1-79.2, and 1.63-6.04\%, respectively, which are better than that, 1.1-11.2, 15.1-29.1, and 0.61-1.89\%, achieved using the fuzzy algorithm, in the cases of voice traffic (CBR), video traffic (VBR), and data traffic (VBR), respectively. Furthermore, simulation results indicate that the proposed GRAMAKA performs best in terms of performance index which consists of throughput and fairness index. The reason why GRAMAKA outperforms other two algorithms is because it offers greater flexibility in the insertion of the slots into the UL-MAP than do the other two approaches.

In the near future, the performance of heterogeneous network which consists of both WiMAX and Long-Term Evolution (LTE) will be investigated by using modified GRAMAKA and other optimization algorithms, where packet delay will be analyzed.

\footnotetext{
Acknowledgements

The authors would like to thank the anonymous reviewers and the editor for their valuable comments. Also, the authors are sincerely grateful to Prof. Tan-Hsu Tan, in the Department of Electrical Engineering, National Taipei University of Technology, Taipei, Taiwan, for his valuable suggestions in refining this article.

\section{Competing interests}

The authors declare that they have no competing interests.

\section{Author details}

'Department of Computer Science and Engineering, Yuan-Ze University, Chung-Li 32003, Taiwan. 'Department of Information Management, University of Kang Ning, Taipei 11485, Taiwan. ${ }^{3}$ Department of Computer and Communication Engineering, Ming-Chuan University, Tao-Yuan 33348, Taiwan.
}

Received: 21 June 2014 Accepted: 27 June 2016 Published online: 15 July 2016

\section{References}

1. IEEE Standard 802.16e-2005, "IEEE Standard for Local and Metropolitan Area Networks - part 16: Air Interface for Fixed and Mobile Broadband Wireless Access Systems. Amendment 2: Physical and Medium Access Control Layers for combined Fixed Mobile Operation in Licensed Bands and Corrigendum 1," 2006

2. IEEE Standard 802.16-2004, "IEEE Standard for Local and Metropolitan Area Networks - Part 16: Air Interface for Fixed Broadband Wireless Access Systems," 2004

3. H. Liu, Y. Jiao, Y. Gao, S. Lin, Performance evaluation of flexible duplex implement based on radio frame selection in LTE heterogeneous network. 22nd International Conference on Telecommunications (ICT), pp. 308-312, 2015

4. H. Pervaiz, L. Musavian, Ni Qiang, Area energy and area spectrum efficiency trade-off in $5 \mathrm{G}$ heterogeneous networks. IEEE International Conference on Communication Workshop (ICCW), pp. 1178-1183, 2015

5. K Sankarasubramaniamand, S Subramanian, A performance study of uplink scheduling in WiMAX network, in International Conference on Recent Trends In Information Technology (ICRTIT), 2012, pp. 377-382

6. P.B. Metre, K.R. Radhika, G. Gowrishankar, wrishankars In Information Technology (ICRTIT)X network(ICCW)G heterogen. International Conferenceon Multimedia Computing and Systems, pp. 562-565, 2012

7. A. Gupta, B.R. Chandavarkar, d B.2.enceon Multimedia Computing and Systems CCW)erogeneous networksworkstworksworksn Multimedia Computing and Sy 7th IEEE International Conference on Industrial and Information Systems (ICIIS), pp. 1-5, 2012

8. A. Sohail, 2012. onal Conference on Industrial and Information Systems (ICllnrtPS in WiMAX Uplink Traffic,f 14th International Conference on Computer Modelling and Simulation (UKSim), pp. 636-639, 2012

9. Z.T. Chou, Y.H. Lin, "Bandwidth Allocation and Recovery for Uplink Access in IEEE 802.16 Broadband Wireless Networks, a IEEE 66thVehicular Technology Conference, pp. 1887-1891, 2007

10. A Bacioccola, C Cicconetti, A Erta, L Lenzini, E Mingozzi, Bandwidth Allocation with Half-Duplex Stations in IEEE 802.16 Wireless Networks, a. IEEE Trans. Mob. Comput. 6(12), 1384-1397 (2007)

11. C.H. Chiang, W.J. Liao, T.H. Liu, Adaptive Downlink/Uplink Bandwidth Allocation in IEEE 802.16 (WiMAX) Wireless Networks: A Cross-Layer Approach. IEEE Global Telecommunications conference, pp. 4775-477, 2007

12. J Deng, Introduction to Grey System Theory. The Journal of Grey System 1, $1-24(1989)$

13. WH Zhang, Handover Decision Using Fuzzy MADM in Heterogeneous Networks. IEEE Wireless Communication and Networking Conference 2, 653-658 (2004)

14. X.H. Xiong, A.B. Ning, M. Liang, A.B. Wang, competitive Decision Algorithm for Multidimensional Knapsack Problem. 2009 International Conference on Management Science and Engineering, ICMSE 2009, pp. 161-167, 2009.

15. T Rasheed, MH Ahmed, OA Dobre, W Jerjawi, M Saad, User pairing in cooperative wireless network coding with network performance optimization. EURASIP J. Wirel. Commun. Netw. 2013, 173 (2013). 2013

16. J. Xing,W. Fan, Z. Lu, A cross-layer scheduling scheme for video streaming based on fuzzy decision-making. 13th IEEE International Conference on Communication Technology (ICCT), pp. 84-87, 2011

17. TM Nguyen, T Yim, Y Jeon, Y Kyung, J Park, QoS-aware dynamic resource allocation for wireless broadband access networks. EURASIP J. Wirel. Commun. Netw. 104, 2014 (2014)

18. Network Simulator 2, available at http://www.isi.edu/nsnam/ns/.

19. J.-Y. Le Boudec and M. Vojnovic,"Perfect Simulation and Stationarity of a Class of Mobility Models," IEEE Infocom 2005, Miami, FL, 2005

20. BJ Hwang, IS Hwang, CW Huang, Frame-based Adaptive Uplink Scheduling Algorithm in OFDMA-based WiMAX Networks. Int. J. Commun. Syst. 25(11), 1381-1397 (2012)

21. BJ Hwang, IS Hwang, CY Chiu, An adaptive ant colony channel-aware slot assignment in OFDMA-based mobile WiMAX networks. Journal of Internet Technology 16(6), 963-975 (2015)

22. J.W. Peng, C.J. Chang, P.L. Tien, A Nonlinear-Predictive QoS-Promoted Dynamic Bandwidth Allocation Scheme for Triple-play Services in Ethernet Passive Optical Networks. IEEE Global Telecommunications conference, pp. 1-5, 2009

23. E Yaacoub, Z Dawy, A survey on uplink resource allocation in OFDMA wireless network. IEEE Commun. Surv. Tutorials 14(2), 322-337 (2012)

24. C.P. Wang, C.Y. Hsu, T.T Tai, Context-based uplink resource allocation for ertPS service in mobile WiMAX networks. 2nd International Conference onConsumer Electronics, Communications and Networks (CECNet), pp. 1735-1739, 2012

25. M. George, R.D. Koilpillai, Fairness-based resource allocation in OFDMA downlink with imperfect CSIT. International Conference on Wireless Communications \& Signal Processing (WCSP), pp. 1-6, 2013 